\title{
Software System and Product Integration Algorithm «Government Project»
}

\section{Ihor Petrovich Zasukha}

Department of Project Management, Kyiv National University of Construction and Architecture, Kyiv, Ukraine

Email address:

igor.zasuha31@gmail.com

\section{To cite this article:}

Ihor Petrovich Zasukha. Software System and Product Integration Algorithm «Government Project». American Journal of Operations Management and Information Systems. Vol. 6, No. 1, 2021, pp. 1-8. doi: 10.11648/j.ajomis.20210601.11

Received: December 28, 2020; Accepted: January 8, 2021; Published: January 22, 2021

\begin{abstract}
Reasonability of the development of new automated system for semantic data integration basing on analysis of advanced IT resource integration tools and methods is described in this article. Software system and product integration algorithm, called as "Government Project", is proposed. This algorithm may be applied for automatic data processing. Its implementation is possible basing on computing system destined to take managerial decisions in the project and project portfolio. Technical result of algorithm application means functional expansion and acceleration of information processing. It is possible to implement this algorithm basing on the automated system which consists of server with software, at least one workstation for input of network-interconnected data. Proposed algorithm allows to create the method to process data for decision-making in the project and project portfolio, to accelerate the parameter calculation process, and to ensure guaranteed availability of all parameters required for managerial decision-making, therefore, to provide technical capacity to improve the quality of made decisions with simultaneous decrease of time required for decision-making. The most successful application of claimed automated data processing method for managerial decision-making is industrial application for decision-making and summarizing the projects using computer equipment.
\end{abstract}

Keywords: Project Management, Project, Information Systems, Software Products, Integration, Algorithm

\section{Introduction}

Existing systems and methods used to transfer planning changes in project management plan may be efficiently applied for selection and control of actions of the contractor involved in the certain project. Nevertheless, they do not cover the issues of checking of reasonability from the point of view of general project development strategy (conformity to the strategic goals) or its successful completion from commercial point of view (investment attractiveness parameters).

Low functional capacities, long time of information processing and low speed of project decision-making are the limitations of technical solution.

Therefore, there is a need to improve computing system and method to manage the project or project portfolio, which ensures resolution of all issues connected with project planning and completion in time due, together with mandatory input and storage of data required for that.

IT development resulted in creation of large number of various heterogeneous information systems (IS), in particular, computer-added design systems (CAD systems) destined to automate business processes, to store personal, reference and other types of data, to maintain statistics and reporting. Such direction as project management is also characterized by availability of different IS and numerous sources of information, eg.: regulatory and reference data storage systems, specialized software for calendar and resource planning. Therefore, the term of information system includes different CAD systems, different database management systems (DBMS), and other automation systems. These systems differ by architecture, access ways, organization of information storage and processing, data models and many other features, nevertheless, in practice it appears that to some extent they are actually similar. Therefore, interoperability and integration are the hottest issues of IS development at the present time [1]. The scope of processed information continues to grow and IT experts have to ensure efficient information exchange among the systems, avoiding at the same time information loss or duplication [2].

Data integration in IS means creation of the common unified 
interface for access to the certain series of heterogenous and independent sources [3]. In other words, information resources from all distributed sources may be available for user from any integration system or from common data access interface. The systems in which these functions are implemented are known as integration systems, and they do not provide to the user or other integrated system the data about sources from which they receive information, the method to get access to them, and features of these sources.

The sources of distributed data may be the common database management systems which operate basing on different approaches: relational, object-oriented, objectrelational; and different systems based on the other technologies. Therefore, provision of access to the data from many heterogenous sources through the common interface actually means creation of unique representation of the complex of all data from multiple independent sources within the framework of one subject domain [11].

The problems in resolution of this task are caused by the fact that information systems are mainly distributed, i.e., they are physically remoted one from another; they are sophisticated software complexes with large number of information units. Besides, they are created basing on different technologies with use of different exchange protocols. These factors are the obstacles in creation of the common unified data warehouse [4].

Traditional methods to resolve this task usually work basing on manually-made relations between the system elements; they are implemented as the software focused on resolution of integration task in each individual case. [13]. Such approach is time-consuming due to the fact that detailed analysis of each system is required to identify the relations and dependences [14]. In addition, structural change of one of the systems, as a rule, results in malfunctions in software complex and requires repeated and time-consuming structural analysis. So, traditional methods do not provide properly unified and flexible solution to create the data integration system. Nevertheless, such methods are usually implemented in practice [15].

Another important task is to ensure interoperability [5]. Interoperability (functional compatibility) means capacity of information system to interact with other systems. Such interaction may have a form of data exchange or federative execution of search requests. [6]. Importance of interoperability problem is determined by the fact that it is highly demanded in the new systems being under development, and in existing IS as well, in order to implement resource and data exchange. This task is closely connected with data integration task, and plays an important role in it [7].

Approaches to ensure interoperability may be provisionally divided in two types: structural and semantic [12]. Structural type means structural coordination of different elements in IS. Semantic type means possibility to establish relations between the senses of elements in information systems. At the present moment main part of existing methods and tools to resolve the problem of interoperability is aimed at structural interoperability without analysis of semantic, i.e., notional system features [3].

Therefore, there is a need to develop the new methods to ensure semantic interoperability of information systems, and to create the new systems for information resource integration - such situation determines the importance of research. Implementation of semantic integration in CAD systems in public project and program management will ensure improvement of general level of decision-making automation, and will simplify the process of search of optimal solutions. It is necessary to develop the new algorithms and software products which could ensure intellectual integration of the systems in common information field. Such software products should be unified, reliable and relatively simple in use.

At the moment one of the most promising integration methods is the method based on use of metadata for description of information resources [8]. This method is closely connected with semantic network technology and its recent and promising direction, linked data technology. This method and technology were taken as the basis for software solution developed in this research, i.e., software ensuring semantic interoperability of the systems and integration of data stored in them.

\section{Research Findings}

The objective of research is to develop the new methods and algorithms for integration of heterogenous sources of information resources in order to improve efficiency of their interaction. New algorithms to ensure interoperability and semantic integration of information systems should be created and substantiated from scientific point of view; the software complex should be developed basing on them [10]. Algorithms should be implemented basing on the research in area of methods and languages used to describe metadata, on the advanced semantic technologies, such as linked data. Software complex should be developed taking in consideration the differences in contemporary platforms and heterogeneity of data sources.

Project management system (project quality management system) includes computer device destined for database storage, which is connected with report formation block, planning block, project implementation management block, budget formation block, resource management block, security block, the block for integration with external systems, quality management block, project changes management block, pending issues management block; each of above-mentioned blocks is connected with all other blocks and with at least one computer device destined to receive and send e-mail messages, and with at least one computer device destined to form user interface in web navigator when the system is connected to the Internet. The system may additionally include the device destined to ensure secure access of users which are out of the limits of system corporate network, basing on request filtering and re-routing mechanism; this device is connected with all above-mentioned blocks and above-mentioned device destined to form user interface in web navigator when the system is 
connected to the Internet.

The invention is in area of computer science, namely, business process automation systems; it may be used, in particular, for project management automation processes.

The following information and analytical system for modelling of rational business system of the company is known: it includes computer device for conceptual description of the company's business, computer device for factor analysis of environment, operator's workstation, computer device for modelling of the business system of operating company, computer device for modelling of optimal structure of the business system of the company, computer device to test business system of the company, computer device for analytical monitoring of business system of the company, and re-engineering computer device. Disadvantages of this system also include limited functional capacities because this system does not ensure control of performance of the modelled business processes due to the absence of blocks with controlling functions in structure of this system.

The following computer-based enterprise management system is known: computer system with processors, data inputoutput devices, memory with databases stored in it, including the block for planning of technical and economic parameters of the enterprise, block for document flow during planning of enterprise parameters, block of accounting and managerial accounting of parameters at the stage of planning, enterprise control and management block, block for document flow during enterprise control and management, block of accounting and managerial accounting for enterprise control and management, block for adjustment of planned enterprise parameters, block for comparison of actual and planned enterprise parameters, and block for adjustment of enterprise document flow. Nevertheless, the functional capacities of this system are limited because this system manages only the certain project of work of individual enterprise due to the use in system structure of above-mentioned blocks featured by the certain limited functional capacities.

The closest analogue of claimed invention is the wellknown project management system described in US patent No. RE 3 8,633, MPK G 06 F 17/60. This system includes computer device destined to store databases, which is connected to report formation block, planning block, project execution management block, budget formation block, resource management block, security block; each of abovementioned blocks is connected with all other blocks and with at least one computer device destined to ensure e-mail message receipt and sending. The system described is featured by limited functional capacities, in particular, it does not ensure quality control in process of project execution, and does not ensure the way to organize interaction among the members of project teams basing on the Internet and appropriate protocol, which is optimal from economic and technical points of view.

For the purpose of claimed invention the project shall mean the obligation to create value based on the project mission, which should be performed during the certain period, within agreed timeframe, resources and operation conditions [9]. For the purpose to implement the project, the group of people is formed; these people are specially trained to resolve the certain tasks according to the project. Each member of project team is assigned the certain function which corresponds to the certain authorities related to the access to information, possibilities to change and amend it. The project may be successfully implemented only in case of resolution of all tasks in time due and with appropriate quality at each stage of project implementation. Project management shall mean the process of planning, organization and control of status of project tasks and resources aimed at achievement of project objectives in time due.

The task to be resolved with use of claimed invention is to create automated project management system which ensures safe, efficient and low-cost interaction of the members of project team taking in consideration the hierarchy, and guarantees full control of project implementation in accordance with the plan.

Technical result of use of proposed solution means expansion of system functional capacities, improvement of its efficiency and reliability.

Above-mentioned task is resolved, and above-mentioned technical result is achieved due to the fact that project management system which includes computer device destined to store databases, connected with report formation block, planning block, project execution management block, budget formation block, resource management block, security block, provided that each of above-mentioned blocks is connected with all other blocks and with at least one computer device destined to ensure e-mail message receipt and sending, includes additionally the block for integration with external systems, quality management block, project change management block, pending issues management block, each of them is connected with each other and abovementioned blocks, with computer device destined to store databases, with at least one computer device destined to ensure e-mail message receipt and sending, and with at least one computer device which is additionally included in the system and destined to form user interface when the system is connected to the Internet. Provided that in order to ensure higher security, the system may additionally include the device destined to ensure secure access of users which are out of the limits of corporate network of the system, basing on request filtering and re-routing mechanism; this device is connected with all above-mentioned blocks and abovementioned device destined to form user interface in web navigator when the system is connected to the Internet.

Inclusion in the system of additional block for integration with external systems, quality management block, project change management block, pending issues management block allows to expand significantly the system functions (eg., to track the presence of claims concerning quality, their change, and existence of problems (pending issues), change of their status), to automate the processes of changes in the projects, and to ensure efficient interaction with external systems.

Inclusion in the system of computer device destined to form user interface in web navigator when the system is connected to the Internet, allows to ensure, with due regard to 
hierarchy, efficient and low-cost interaction among the members of project team.

Invention is represented at the figure showing the system block diagram (figure 1).

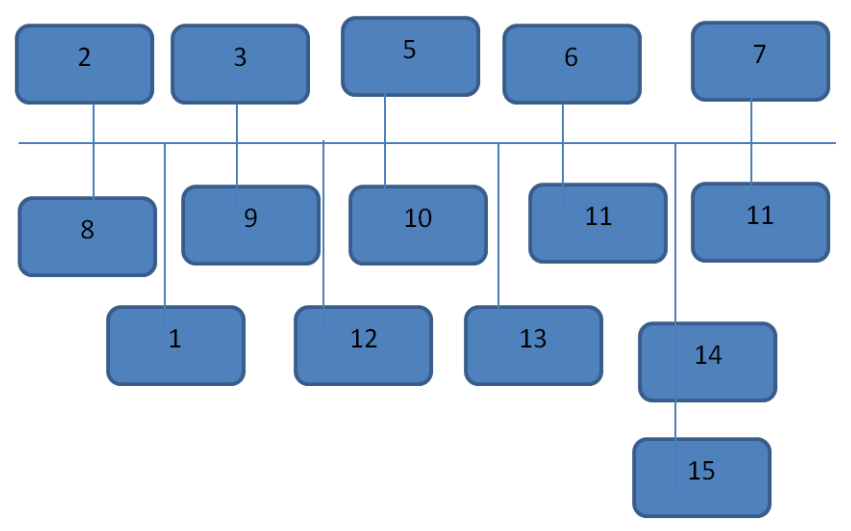

Figure 1. Block Diagram of the Government Project, Project Management System.

Project management system includes computer device 1 destined to store databases, including databases of documents of any format, report formation block 2, planning block 3, project execution management block 4 , budget formation block 5, resources management block 6 , security block 7 , block for integration with external systems 8 , quality management block 9, project change management block 10, pending issues management block 11, computer device 12 destined to ensure receipt and sending of e-mail messages, computer device 13 destined to form user interface in web navigator when the system is connected to the Internet, computer device 14 destined to ensure secure access of the users being out of the limits of system corporate network. The second computer device 15 may be additionally connected to the system - it is destined to form user interface in web navigator when the system is connected to the Internet.

Computer device 1 destined to store databases is connected with report formation block 2, planning block 3, project execution management block 4, project budget formation block 5, resource management block 6 , security block 7 , block for integration with external systems 8 , quality management block 9, project change management block 10, pending issues management block 11. All above-mentioned blocks are connected with each other and with computer device 12 which is destined to ensure e-mail message sending and receipt, with computer device 13 destined to form user interface in web navigator when the system is connected to the Internet, and with computer device 14 destined to ensure secure access of users being out of the limits of system corporate network, which is connected to the computer device 15 destined to form user interface when the system is connected to the Internet.

Computer device 1 destined to store databases which contain project information, may be implemented basing on computer, eg., with one CPU Intel Pentium IV, clock speed at least 2,2 GHz, random access memory at least $1 \mathrm{~GB}$ and disk subsystem with capacity at least $5 \mathrm{~Gb}$, with use, eg., of industrial database server Oracle $10 \mathrm{~g}$ or Microsoft SQL Server 2000. The structure of this computer device 1 may include the memory block destined to store databases with documents represented in any format, eg., technical documents in the project, user manuals, etc. The special software, eg., Lotus Notes or Microsoft SharePoint, may be used to store the documents.

Report formation block 2 forms the reports about project status according to the enterprise project management rules. Provided that the reports may have the pre-set form, or the form may be determined by users with appropriate authorities, eg., the block forms the following reports: "Report on the works completed in the project", "Report on the works completed with distribution by customers", "Report on working time consumption in the project", "Report on working time consumption with distribution by employees", etc.

Block 2 may be implemented basing on computer with two CPU Intel Pentium IV, clock speed at least 2,2 GHz, random access memory at least $1 \mathrm{~GB}$ and disk subsystem with capacity at least $5 \mathrm{~Gb}$, with use of special software, eg. Crystal Reports or MS Office.

Planning block 3 ensures performance of the following functions:

1. Project plan review;

2. Project plan editing (creation/change/change cancellation, replacement of task executives);

3. View of individual task list (the tasks to be performed by employee).

Planning block 3 allows to:

1. Assign resources available for project planning;

2. Change status of performance of individual tasks in the project;

3. Approve changes and amendments in project tasks made by project participants;

4. Save changed project plan in appropriate database;

5. Transfer data to form the relevant reports.

Block 3 may be implemented basing on computer with one CPU Intel Pentium IV, clock speed at least 2,2 GHz, random access memory at least $1 \mathrm{~GB}$ and disk subsystem with capacity at least $5 \mathrm{~Gb}$, with use of special software, eg. Microsoft Project Server 2003, SAP project System or Primavera P3E.

Project execution management block 4 is destined to issue orders and control their execution. Orders are issued by formation of the relevant instructions and their sending to the addresses. Execution is controlled by fixation of receipt of the certain data sets or their absence at the certain moment of time.

Block 4 may be implemented basing on computer with one CPU Intel Pentium IV, clock speed at least 2,2 GHz, random access memory at least $1 \mathrm{~GB}$, and disk subsystem with capacity at least $5 \mathrm{~Gb}$, with use of special software, eg. SAP xRPM (Resource and Program Management) or Microsoft Project Server.

Budget formation block 5 ensures for authorized user the 
possibility to manage (create, view, change budget versions) the project budget formed basing on specially prepared data. In line with that the block 5 provides for user access to the information about all approved budget versions, and about current budget execution (planned-actual) with comparison of data with project plans. Block 5 ensures for project members determination of rules for sending notifications related to the budget/budget version processing in the project. Block 5 forms the data for report about project budget status.

Block 5 may be implemented basing on computer with one CPU Intel Pentium IV, clock speed at least 2,2 GHz, random access memory at least $1 \mathrm{~GB}$, and disk subsystem with capacity at least $5 \mathrm{~Gb}$, with use of special software, eg. MS Excel tables or Microsoft Project Server 2003.

Resource management block 6 performs the following functions: creation and change of the list of user's accounts of project members; determination of functions of each project member, granting of user's authorities according to the user's function; analysis of workload on project member according to the assigned tasks, etc.

Block 6 may be implemented basing on computer with one CPU Intel Pentium IV, clock speed at least 2,2 GHz, random access memory at least $1 \mathrm{~GB}$, and disk subsystem with capacity at least $5 \mathrm{~Gb}$, with use of special software, eg. Microsoft Project Server, Microsoft Exchange Server.

Security block 7 implements the mechanism of roles and functions (eg., project coordinator, manager, team manager, project participant, administrator, customer), ensuring users' hierarchy and limitation of users' access to the system functions.

Block 7 may be implemented basing on computer with one CPU Intel Pentium IV, clock speed at least 2,2 GHz, random access memory at least $1 \mathrm{~GB}$, and disk subsystem with capacity at least $5 \mathrm{~Gb}$, with use of special software, eg. Microsoft Windows 2000 Server.

Block for integration with external systems 8 is destined to ensure interaction with external structures, eg., with staff management system, etc.

Block 8 may be implemented basing on computer with two CPUs Intel Pentium IV, clock speed at least 2,2 GHz, random access memory at least $1 \mathrm{~GB}$, and disk subsystem with capacity at least $5 \mathrm{~Gb}$, with use of special software, eg. Microsoft BizTalk Sever or SAP XI.

Quality management block 9 ensures claim management, provided that users should have a possibility to create/change/cancel claims according to authorities fixed in the system, and according to the project claim processing rules. This block provides a possibility to search claims in the system, to set the number and sequence of presentation of displayed claim parameters in resulting list, and to set project claim processing rules.

Block 9 may be implemented basing on computer with two CPUs Intel Pentium IV, clock speed at least 2,2 GHz, random access memory at least $1 \mathrm{~GB}$, and disk subsystem with capacity at least $5 \mathrm{~Gb}$, with use of special software, eg. Rational Clear Quest.

Project change management block 10 ensures a possibility to make changes (create, view, edit, cancel) in the project, and to search project changes. Block 10 allows the project members to determine the rules for sending notifications related to the project change request processing, and to export data connected with change request from the database to MS Office document template.

Block 10 may be implemented basing on computer with one CPU Intel Pentium IV, clock speed at least 2,2 GHz, random access memory at least $1 \mathrm{~GB}$, and disk subsystem with capacity at least $5 \mathrm{~Gb}$, with use of special software, eg. Rational Requisite Pro or Rational Clear Quest.

Pending issues management block 11 ensures management of pending issues (unresolved problems). Project pending issue shall mean any functional, technical, organizational or business-related issue which occurs in process of project implementation and should be resolved to ensure normal progress of works.

Users have a possibility to create/change/cancel pending issues according to authorities fixed in the system and project pending issues processing rules. Block 11 provides a possibility to search pending issues in the system, an option to set number and sequence of presentation in the resulting list of displayed parameters of pending issues, and the rules of project pending issues processing. Block 11 forms the necessary data sets to prepare the relevant reports.

Block 11 may be implemented basing on computer with one CPU Intel Pentium IV, clock speed at least 2,2 GHz, random access memory at least $1 \mathrm{~GB}$ and disk subsystem with capacity at least $5 \mathrm{~Gb}$, with use of special software, eg. Microsoft SharePoint or Rational Clear Quest.

Computer device 12 is destined to ensure receipt and sending by e-mail to the agents of notifications related to the different system events according to the certain rules. It may be implemented basing on computer with one CPU Intel Pentium IV, clock speed at least 2,2 GHz, random access memory at least $1 \mathrm{~GB}$ and disk subsystem with capacity at least $5 \mathrm{~Gb}$, with use of special software, eg. SMTP server.

Computer devices 13 and 15 destined to form user interface in web navigator when the system is connected to the Internet, may be implemented basing on computer with one CPU Intel Pentium IV, clock speed at least 2,2 GHz, random access memory at least $1 \mathrm{~GB}$ and disk subsystem with capacity at least $5 \mathrm{~Gb}$, with use of special software, eg. Microsoft Internet Information Server (US).

Computer device 14 destined to ensure secure access of users being out of the limits of corporate system network, may be implemented basing on the router, eg., Cisco PIX 515 or Checkpoint FireWall 1, with external traffic control function and protection of internal corporate network from external intrusions (FireWall).

System operation may be described using as an example the project plan formation and management of its execution.

Proposal to start the project is registered. For this purpose the written message with proposal to start the project work is prepared (special form is filled) at the computer of project initiator (not shown at the figure), using the special software. Data corresponding to the message formed are transferred by 
e-mail to the system in form of the relevant signals; these data are received by computer device 12 . Computer device 12 identifies the signal received as the data corresponding to proposal to start the project, and sends it to e-mail of the person making the decision about possibility to start the project.

The person who received proposal to start the project, accepts or rejects this proposal. In case of reject the system does not work anymore for this project; if the project was accepted, the system operation continues.

When the decision to start the project work is adopted, the project priority is determined and project manager is nominated. In this case the message about nomination of the certain person to act as project manager is formed. This message in form of signal corresponding to the abovementioned data is sent to the computer device 12 which identifies the signal received as message for the certain person and forms e-mail message. This message in form of the relevant signals is sent to e-mail of specialist nominated to act as project manager. Data corresponding to proposal to start the project, and data determining priority assigned to the project, are transferred from computer device 12 to the computer device 1 , where they are registered in the relevant database.

Project manager prepares project plan, determines the tasks required in order to implement the project, fixes project stages and completion deadlines. Project manager sends the plan formed with due regard to the system requirements, in form of the certain data set including parameters which describe the project, to the computer device 1.

Data received by computer device 1 are identified as the data corresponding to project implementation plan, so, they are recorded in the relevant database.

The data describing initial requirements for project completion results, risk factor assessment and other information required to implement the project are also recorded in the relevant database. Before recording the data should be processed and prepared accordingly.

When the tasks, execution of which is required for project implementation, are determined, the project manager forms the project team structure and assigns the certain function to each team member. Project manager forms the relevant message and transfers it in form of the certain data set to computer device 1 for its record in the relevant database.

Thereafter, the project manager should select actual executives according to the functions in project team. For this purpose he/she creates request in form of the relevant data set and sends it through the block for integration with external systems 8 to the staff management system (not shown at the figure). In response to the message received the staff management system forms the list of possible project team members and sends it in form of the relevant data set to the block for integration with external systems 8 . From this block the data are sent to computer device 1 where they are recorded in relevant database, and to computer device 12, from which they are transferred to project manager in form of message consisting of the relevant data set.
Then the project manager coordinates allocation of specialists to the project team with managers of their resource departments. Taking the decision to allocate specialists to the project team, the managers of resource departments take in consideration information available in the system concerning their current and future workload. If necessary, negotiations and coordination have place.

Negotiations and coordination may be performed by forming of the relevant messages and message exchange among the persons who approve the relevant decisions.

In process of plan preparation and approval the authorized persons may introduce changes in it with use of planning block 3. In this case the authorized user logs to the system through computer device 13 or 15 . Security block 7 checks the user's authorities according to the method stipulated in the rules, with use of well-known software (eg., by user login and password verification, by input of biometric data and their comparison with data recorded in the relevant database, or with use of hardware key). When the user's authorities are confirmed, he/she forms the command (eg., by selection of appropriate pictogram) to perform the certain actions. The command in form of relevant data set is transferred to the planning block 3 where it is processed with use of special software. As a result the planning block 3 operates in accordance with the command received. Eg., user may view the project plan or edit it.

Budget plan is also formed in process of plan preparation and approval. For this purpose the budget formation block 5 is used. According to the commands received by abovementioned block 5 in form of the relevant data sets, block 5 requests the relevant database stored at computer device 1 , or, using block 8 , requests external systems to provide initial data (eg., requests external staff management system to provide the data about costs connected with project members) required to calculate the budget parameters. Block 5 processes these data according to the request and sends them to computer device 12 , from which they are transferred to user in form of message consisting of the relevant data set.

When project plan and project team structure are approved, the project implementation is initiated, the project receives the status of approved and being at the stage of implementation.

Project execution management block 4 reads from the relevant databases the data (tasks, deadlines, etc.) from the project plan and forms the data sets corresponding to the instructions concerning project execution. It sends these data to computer device 12, where they are identified as the messages for project team members, and sent to the relevant addresses.

In process of project implementation the executives report on progress in execution of the tasks. Users provide to system the data describing the status of task execution (percent of execution, deadlines, etc.), and transfer them to the project execution management block 4 , and to computer device 1, from which the data are sent as necessary (according to the rules) to the report formation block 2 . 
During this process the project execution management block 4 fixes receipt or absence at the certain moment of time of the relevant data sets, forms the relevant notification in form of the relevant data sets, and transfers them to the computer device 12. The data received by computer device 12 are identified as the messages for project team members, and sent to the relevant addresses. Report formation block 12 forms the reports in accordance with pre-set form or the form set by users with appropriate authorities. Reports formed in the block 2 are transferred in form of the relevant data sets to computer device 12 , where the data received are identified as the messages for project team members, and sent to the relevant addresses.

In process of project execution the executives may face with the problems which they cannot resolve themselves. In this case executive forms the message describing the problem (pending issue), and transfers it to the system in form of the relevant data set. These data are transferred in the system to computer device 1 and recorded in the relevant database. During this process the pending issues management block 11 forms notification about receipt of the pending issue and transfers the relevant data sets to computer device 12, where the data received are identified as the messages for project team members and sent to the relevant addresses. Due to the pending issues management block 11 the authorized users, giving the relevant commands, have the possibility to create/change/cancel pending issues according to the authorities fixed in the system and project pending issue processing rules, to search pending issues in the system, and the possibility to set the number and sequence of presentation of pending issue parameters displayed in the resulting list, and the rules for project pending issues processing. Block 11 forms the data sets required to create the relevant reports, and sends them to the report formation block 2 .

To ensure control of project execution quality, conformity of project progress to the project implementation requirements is checked. For this purpose the quality management block 9 is used. Using the quality management block, users form descriptions of claims and send them in form of the relevant data sets to computer device 1 where they are recorded in the relevant database. Due to the certain commands provided in software used, the authorized users have a possibility, using quality management block 9, to create/change/cancel claims according to their authorities fixed in the system, and according to the project claim processing rules, and to search the claims.

In process of project implementation the need to make changes in project plan may occur. In case of such need the authorized user, using the project change management block 10 , by selection of appropriate command makes changes (creation, view, editing, cancelation) in the project. Project change management block 10 forms the data set corresponding to the changes and transfers them to computer device 1, where these data are recorded to the relevant database; these data are also sent to the computer device 12 where they are identified as the messages for project team members, and sent to the relevant addresses.

Information about project execution status is stored in the system database for its further analysis aimed at improvement of project implementation quality in future.

\section{Conclusion}

Therefore, the invention allows to create the method of data processing for decision-making in the project and portfolio of projects, contributing in such a way to the automation of data accounting, accelerating the process of parameters calculation, and ensuring availability of all parameters required to take managerial decisions, thus, ensuring a technical capacity to improve the quality of taken decisions with simultaneous decrease of time required for decision-making.

The claimed method makes it possible to expand the functional capacities, to decrease time needed for managerial decision-making, to accelerate information processing and to improve responsiveness in decision-making on the project issues.

The claimed method of automated data processing for managerial decision-making is the most successful in industrial applications for decision-making and preparation of project conclusions with use of computer hardware.

\section{References}

[1] Batovrin V. K., Huliaev Yu. V., Oleinikov A. Ya. Ensuring Interoperability - the Main Trend in Open Systems // Information Technologies and Computing Systems - 2009. No. 5. - pgs. 7-15.

[2] Kohalovskyi M. R. Advanced Information System Technologies. - Moscow: IT-Economy. - 2003. - 288 pgs.

[3] Mylhailov I. S. Metamodel-Based Mathematical Support and Software for Structural and Semantic Interoperability of Information Systems: thesis of candidate of technical sciences. - NIU MEI.-2003 [Digital resource]. - Access mode: http://www.dissercat.com/content/issledovanie-i-razrabotkametoda-obespecheniya-strukturnoi-interoperabelnostiinformatsionny, private. Language: Russian (request date 15.12.2020).

[4] Novitskyi A. V. Review of the Certain Directions in Integration of Heterogenous Resources in E-Libraries // The works of $11^{\text {th }}$ Russian Scientific Conference "E-Libraries: Advanced Methods and Technologies, Digital Collections" RCDL'2009, Petrozavodsk, Russia. - 2009. - pgs. 350-356

[5] Rajabifard Abbas. Critical issues in global geographic information management with a detailed focused on Data Integration and Interoperability of Systems and Data // Scoping Paper for the 2nd Preparatory Meeting of the Proposed UN Committee on Global Geographic Information Management. New York, USA, May 10-11, 2010. - pgs. 1-14.

[6] Ke-Qing He, Jian Wang, Peng Liang. Semantic Interoperability Aggregation in Service Requirements Refinement // Journal of Computer Science and Technology JCST, v. 25, No. 6. - 2010. - pgs. 1103-1117. 
[7] Ruokolainen Toni. Modelling Framework for Interoperability Management in Collaborative Computing Environments // Department of Computer Science, Series of Publications Report, Helsinki, June 2009.

[8] Polotniuk I. S. Metadata as the Basis for Integration. Journal of Computer and Information Technologies. - 2005. - No. 12.

[9] Bushuev S. D., Bushueva N. S., Babaev I. A., Iakovenko V. B., Hrysha Ye. V., Dziuba S. V., Voitenko A. S. Creative Program and Project Management Technologies: Monograph. - K.: Summit-Kniha, 2010. - 768 pgs.: il.

[10] Semerhanov I. A., Vargin G. V., Muromtsev D. I. Integration of computer systems with the use of ontology // 12th Conference of Open Innovations Association FRUCT. Oulu, Finland, November 5-9, 2012.
[11] Rodriguez-Muro Mariano, Diego Calvanese. Dependencies: Making Ontology Based Data Access Work. In AMW. - 2011.

[12] Sangsoo S. Ontology-based semantic integration of heterogeneous information sources: thesis of doctor of technical sciences. - University of Southern California, 2008.

[13] Maniraj V., Dr. Sivakumar R. Ontology Languages - A Review // International Journal of Computer Theory and Engineering, v. 2, No. 6. - 2010. - Pgs. 887-891.

[14] Rahm Erhard, Bernstein Philip A. A survey of approaches to automatic schema matching // Proceedings of the VLDB Endowment, v. 10, No. 4. - 2001. - Pgs. 334-350.

[15] Rahm Erhard, Bernstein Philip A., Madhavan Jayant Generic Schema Matching, Ten Years Later // Proceedings of the VLDB Endowment, v. 10, No. 11. - 2011. - Pgs. 695-701. 